\title{
Patterns of Flexible Nanotubes Formed by Liquid-Ordered and Liquid-Disordered Membranes
}

Yonggang Liu, ${ }^{\dagger \dagger}$ Jaime Agudo-Canalejo, ${ }^{\dagger}$ Andrea Grafmüller, ${ }^{\dagger}$ Rumiana Dimova, and Reinhard Lipowsky, ${ }^{*}$

${ }^{\dagger}$ Theory \& Biosystems, Max Planck Institute of Colloids and Interfaces, 14424 Potsdam, Germany

${ }^{\ddagger}$ State Key Laboratory of Polymer Physics and Chemistry, Changchun Institute of Applied Chemistry, Chinese Academy of Sciences, 130022 Changchun, China

\section{Supporting Information}

ABSTRACT: Biological membranes form both intra- and intercellular nanotubes that are used for molecular sorting within single cells and for long-distance connections between different cells. Such nanotubes can also develop from synthetic lipid bilayers in their fluid state. Each nanotube has a large area-to-volume ratio and stably encloses a water channel that is thereby shielded from its surroundings. The tubes are rather flexible and can easily change both their length and their conformation. Here, we study

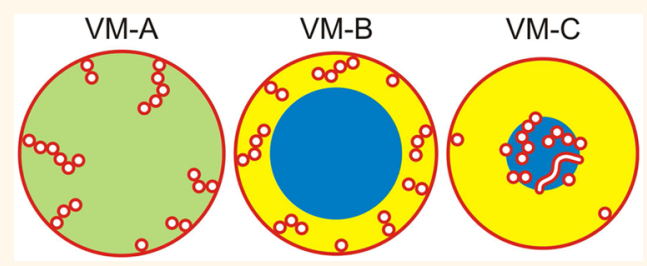
nanotubes formed by liquid-ordered (Lo) and liquid-disordered (Ld) membranes with three lipid components exposed to aqueous mixtures of two polymers, polyethylene glycol (PEG) and dextran. Both types of membranes form striking patterns of nanotubes when we reduce the volume of giant vesicles by osmotic deflation, thereby exposing the two bilayer leaflets of the membranes to polymer solutions of different composition. With decreasing volume, three different patterns are observed corresponding to three distinct vesicle morphologies that reflect the interplay of spontaneous curvature and aqueous phase separation. We show that tube nucleation and growth is governed by two kinetic pathways and that the tubes undergo a novel shape transformation from necklace-like to cylindrical tubes at a certain critical tube length. We deduce the spontaneous curvature generated by the membrane-polymer interactions from the observed vesicle morphologies using three different and independent methods of image analysis. The spontaneous curvature of the $\mathrm{Ld}$ membranes is found to be 4.7 times larger than that of the Lo membranes. We also show that these curvatures are generated by weak PEG adsorption onto the membranes, with a binding affinity of about $1.6 k_{\mathrm{B}} T$ per chain. In this way, our study provides a direct connection between nanoscopic membrane shapes and molecular interactions. Our approach is rather general and can be applied to many other systems of interest such as polymersomes or membrane-bound proteins and peptides.

KEYWORDS: membranes and vesicles, aqueous polymer solutions, membrane nanotubes, tube nucleation and growth, bilayer asymmetry, spontaneous curvature, PEG adsorption

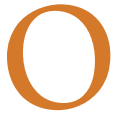
ne important function of biological membranes is to partition space into separate compartments. Particularly interesting compartments are provided by membrane nanotubes which represent highly curved membrane structures, have a large area-to-volume ratio, thereby enhancing membrane-dependent processes, and stably enclose thin water channels which are well-separated and shielded from their surroundings. Intracellular nanotubes are ubiquitous structural elements of many membrane-bound organelles such as the endoplasmic reticulum, the Golgi, the endosomal network, and mitochondria. ${ }^{1-3}$ These nanotubes connect distant parts of the cell and are used for molecular sorting, signaling, and transport. Intercellular (or "tunneling") nanotubes between two or more cells provide long-distance connections for cell-cell communication, intercellular transport, and virus infections. ${ }^{4-6}$
Synthetic nanotubes formed by lipid or polymer bilayers have been studied in the context of bilayer asymmetry and spontaneous curvature, ${ }^{7,8}$ lipid and protein sorting, ${ }^{9,10}$ cellular delivery of drugs and other chemical agents, ${ }^{11}$ and as templates for rigid "nanowires". 12

Biological and biomimetic nanotubes are formed by fluid membranes which have a stable bilayer structure on the molecular scale but are highly flexible on the nanoscopic scale. The fluidity also allows fast lateral transport of molecules along the membranes, easy changes in tube length and conformation, as well as the formation of intramembrane domains and rafts.

Received: August 27, 2015

Accepted: November 20, 2015

Published: November 20, 2015 
Here, we study giant unilamellar vesicles of three-component lipid bilayers. We investigate and compare two different lipid compositions that form a liquid-ordered (Lo) and a liquiddisordered ( $\mathrm{Ld}$ ) phase. The vesicles enclose aqueous polymer mixtures of dextran and polyethylene glycol (PEG), an aqueous two-phase system that has been frequently used in biochemical analysis and biotechnology ${ }^{13}$ to separate and purify biomolecules, cell organelles, and cell membranes. The lipid membranes form striking patterns of nanotubes within the giant vesicles when we deflate these vesicles osmotically, thereby exposing the two bilayer leaflets to polymer solutions of different composition. Three types of nanotube patterns can be distinguished corresponding to three different vesicle morphologies as shown schematically in Figure 1.

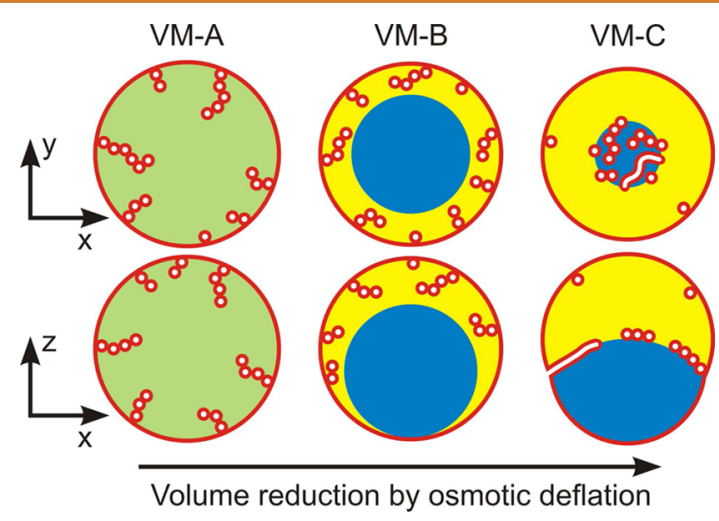

Figure 1. Three nanotube patterns corresponding to the distinct vesicle morphologies VM-A, VM-B, and VM-C observed along the deflation path: Schematic views of horizontal $x y$-scans (top row) and of vertical $x z$-scans (bottom row) across the deflated vesicles. In all cases, the tubes are filled with external medium (white). For the VM-A morphology, the interior polymer solution is uniform (green), whereas it is phase separated (blue-yellow) for the morphologies VM-B and VM-C, with complete and partial wetting, respectively, of the membrane by the PEG-rich aqueous phase (yellow). For the VM-B morphology, the nanotubes explore the whole PEG-rich (yellow) droplet but stay away from the dextranrich (blue) one. For the VM-C morphology, the nanotubes adhere to the interface between the two aqueous droplets forming a thin and crowded layer along this interface. The different nanotube patterns can be most easily visualized by three-dimensional confocal scans of the vesicles, see Supporting Movies.

The theoretical analysis of these observations reveals that the tubes are nucleated from small buds which subsequently grow into necklace-like tubes. The necklaces are further extended until they transform into cylindrical tubes when their length exceeds a certain critical value. The critical length can be reduced by the adhesion of the nanotubes to interfaces. These morphological features are universal and apply to the deflationinduced formation of membrane nanotubes in general.

The magnitude of the bilayer asymmetry, arising from the exposure of the two leaflets to different polymer concentrations, is quantitatively described by the spontaneous curvature of the membranes. We introduce three different and independent methods of image analysis to deduce this curvature from the observed vesicle morphologies. All three methods give very consistent results. The spontaneous curvature $m$ of the $\mathrm{Ld}$ and Lo membranes is found to be $m_{\mathrm{Ld}} \simeq-1 /(125 \mathrm{~nm})$ and $m_{\mathrm{Lo}} \simeq$ $-1 /(600 \mathrm{~nm})$ over a certain range of polymer concentrations. Using atomistic molecular dynamics simulations, we also elucidate the molecular mechanism for the spontaneous curvatures obtained here and conclude that these curvatures are generated by the weak adsorption of PEG chains onto the membranes, with a binding free energy of about $4 \mathrm{~kJ} / \mathrm{mol}$ or 1.6 $k_{\mathrm{B}} T$ per chain, and that the curvature ratio $m_{\mathrm{Ld}} / m_{\mathrm{Lo}} \simeq 4.7$ is approximately equal to the ratio $\kappa_{\mathrm{Lo}} / \kappa_{\mathrm{Ld}}$ of the corresponding bending rigidities. Our approach to deduce the spontaneous curvature from the observed tubulation process is quite general and can be extended to other membrane systems of interest. Two examples are provided by polymer bilayers or polymersomes ${ }^{1,14-16}$ and by the adsorption of proteins ${ }^{17,18}$ or peptides $^{19}$ onto lipid membranes. Accurate estimates for the spontaneous curvature are also crucial in order to determine the critical particle sizes for the engulfment of nanoparticles by membranes and vesicles. ${ }^{20}$

\section{RESULTS AND DISCUSSION}

Tubulation of Vesicles Induced by Osmotic Deflation. Giant unilamellar vesicles were prepared from ternary lipid mixtures of dioleoylphosphatidylcholine (DOPC), dipalmitoylphosphatidylcholine (DPPC), and cholesterol, see Methods. The vesicles were formed in aqueous polymer mixtures of PEG and dextran, which undergo phase separation into two aqueous phases, a PEG-rich and a dextran-rich phase, when the polymer weight fractions exceed a few weight percent ${ }^{13,21,22}$ (Figure 2a,b). Two types of lipid compositions were studied corresponding to a flexible $\mathrm{Ld}$ and a more rigid Lo membrane. ${ }^{23-25}$ The Ld membranes were fluorescently labeled by a red dye, the Lo membranes by a green one (Figure $2 \mathrm{c}, \mathrm{d}$ and Supporting Information Movies).

Our experiments started with spherical vesicles that enclosed a homogeneous polymer solution (composition 0 in Figure $2 b$ ). These vesicles were then deflated osmotically by using exterior solutions that contained fixed weight fractions of the two polymers but an increasing amount of sucrose. The subsequent deflation steps are depicted in Figure $2 b$ and described in more detail in Table S1. After the first deflation step, the interior polymer solution still formed a uniform aqueous phase (composition 1), corresponding to the VM-A morphology in Figure 1. After the second and all subsequent deflation steps, the interior solution underwent phase separation into a PEGrich and a dextran-rich phase which formed two aqueous droplets within the vesicles. The shapes of these droplets could be directly imaged by differential interference contrast and fluorescence microscopy, see Figure S1. The PEG-rich droplet was always in contact with the membranes, whereas the dextran-rich droplet came into contact with the $\mathrm{Ld}$ and Lo membranes only after the third and fourth deflation step, respectively. Therefore, the $\mathrm{Ld}$ membranes were completely wetted by the PEG-rich phase for composition 2, while the Lo membranes exhibited the same type of wetting for compositions 2 and 3, corresponding to the VM-B morphology in Figure 1. Subsequent deflation steps led to the VM-C morphology with partial membrane wetting and nonzero contact angles.

Each osmotic deflation step reduces the vesicle volume and, thus, increases the area-to-volume ratio of the vesicle. In the absence of polymers, vesicles undergo smooth shape transformations from spherical toward prolate shapes as studied previously $^{26,27}$ for one-component membranes. In contrast to such conventional shape transformations, the vesicles studied here still assumed a spherical shape after the initial deflation steps, both for the flexible Ld membranes (Figure 2c) and for 

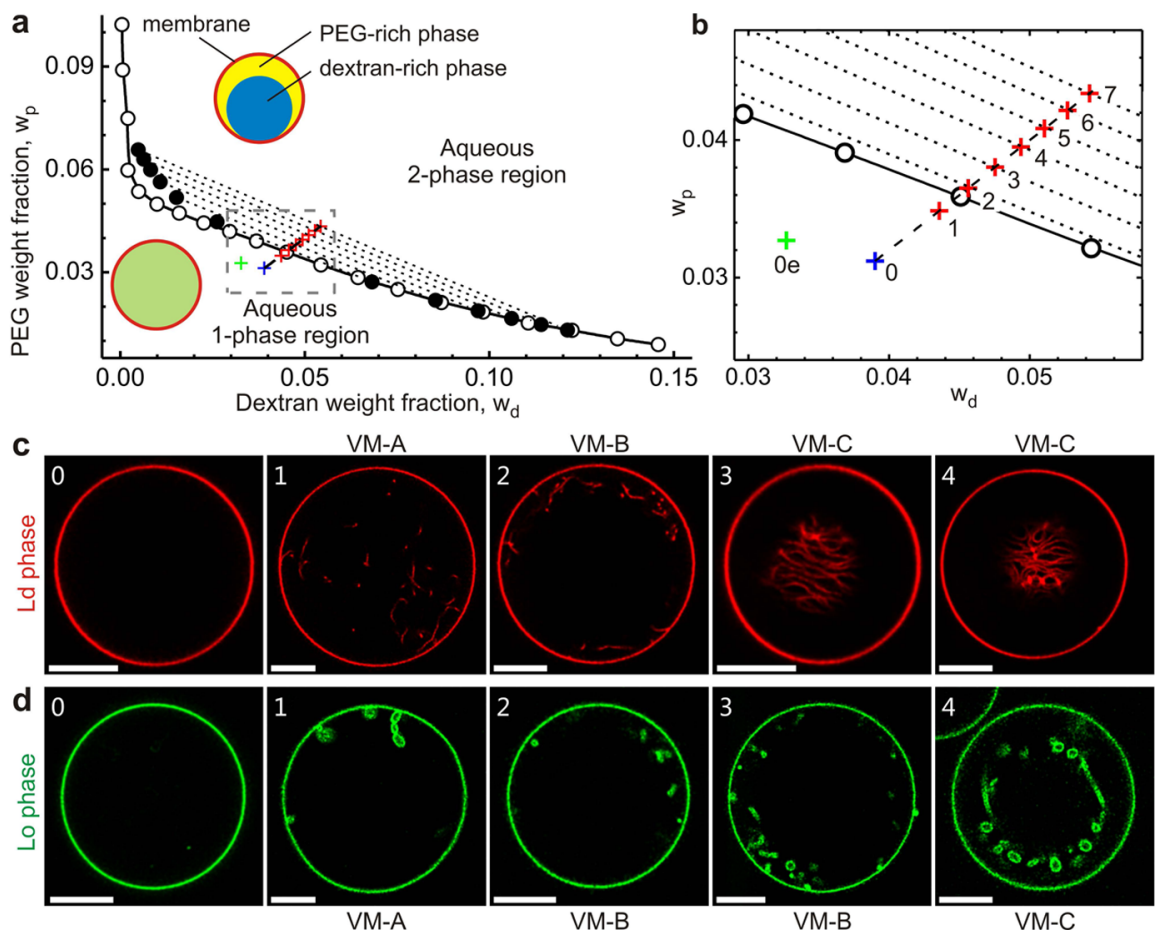

Figure 2. Osmotic deflation and tubulation of vesicles in aqueous PEG-dextran solutions. (a) High-precision phase diagram of aqueous PEG-dextran solution close to its critical demixing point at $24^{\circ} \mathrm{C}$ with dotted tie lines and dashed deflation trajectory. (b) Enlarged region of phase diagram around the deflation trajectory which starts from the initial polymer composition 0 (blue cross) within the vesicle and the composition 0e (green cross) of the isotonic external medium. The vesicle is then osmotically deflated in a stepwise manner which leads to the interior compositions 1-7 (red crosses), see Table S1 for more details. (c) Confocal $x y$-scans of vesicles bounded by an Ld membrane (red) at the deflation steps $0-4$. Apart from the initial vesicle 0 , all deflated vesicles formed membrane nanotubes pointing into the vesicle interior. The thickness of the $\mathrm{Ld}$ tubes was below optical resolution. More detailed views of the three-dimensional morphologies of the vesicles are provided in the Movies $\mathrm{Ld} \_1, \mathrm{Ld} \_2$, and Ld_4. (d) Confocal $x y$-scans of vesicles bounded by an Lo membrane (green) at the deflation points 0-4. The deflated vesicles in panels 1-4 again form nanotubes; the corresponding three-dimensional scans are shown in the Movies Lo_1, Lo_2, and Lo_4. The thickness of the Lo tubes was above optical resolution. The scale bars are $10 \mu \mathrm{m}$ in all confocal images. The confocal $x z$-scans of the vesicles in panels $(\mathrm{c})$ and $(\mathrm{d})$ are displayed in Figure S2.
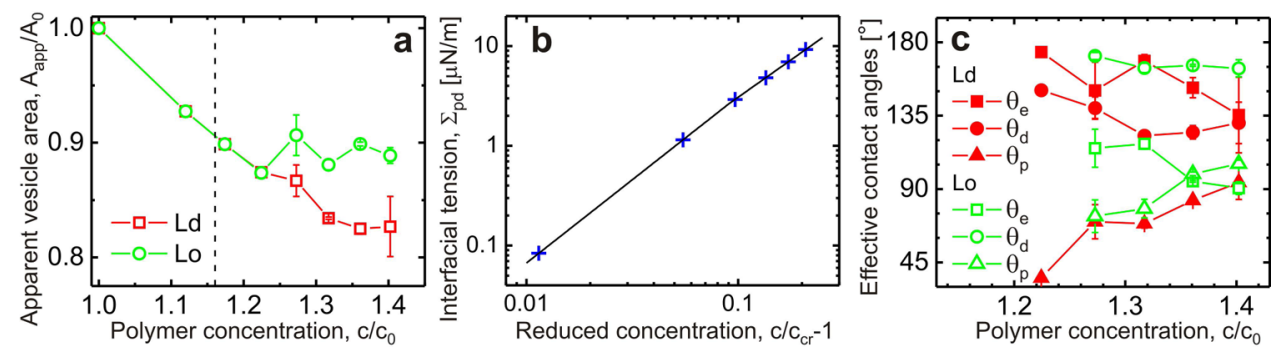

Figure 3. Variation of apparent vesicle area, interfacial tension, and contact angles with osmotic deflation which leads to an increase in the total polymer concentration $c$ within the vesicles. (a) Apparent membrane area of large mother vesicles which decreases monotically during the first three deflation steps both for the Ld and the Lo membranes. The concentration $c_{0}=0.0714 \mathrm{~g} / \mathrm{cm}^{3}$ represents the initial polymer concentration before deflation; the vertical dashed line corresponds to the critical concentration $c_{\mathrm{cr}}=1.161 c_{0}$. (b) Interfacial tension $\Sigma_{\text {pd }}$ of the pd interface (p, PEG-rich phase; d, dextran-rich phase) between the PEG-rich and dextran-rich aqueous phases, data (blue crosses) and linear fit (solid black line). The tension vanishes at the critical concentration and increases monotonically for $c>c_{\mathrm{cr}}$. (c) Effective contact angles $\theta_{\mathrm{p}}, \boldsymbol{\theta}_{\mathrm{d}}$, and $\boldsymbol{\theta}_{\mathrm{e}}$ between the pd interface and the two membrane segments as defined in Figure S3. The contact angle $\theta_{\mathrm{p}}$ vanishes at the concentration $c_{\mathrm{wt}}$ of the complete-to-partial wetting transition with $1.174<c_{\mathrm{wt}} / c_{0}<1.224$ for the Ld membranes (red data) and $1.224<c_{\mathrm{wt}} / c_{0}$ $<1.273$ for the Lo membranes (green data).

the more rigid Lo membranes (Figure 2d). However, because the vesicle volume was reduced by the deflation, the vesicle shapes had an apparent area $A_{\text {app }}$ that was smaller than the initial vesicle area $A_{0}$. As shown in Figure 3a, this apparent area decreased by about $7 \%, 3 \%$, and $2.5 \%$ during the first, second, and third deflation step, respectively. The missing membrane area, $A=A_{0}-A_{\text {app}}$, was stored in nanotubes that protruded into the vesicle interior.
This tube formation was observed for both types of membranes and for all three vesicle morphologies (Figure $2 \mathrm{c}, \mathrm{d})$. For the VM-A and VM-B morphologies, the tubes underwent strong thermally excited undulations and we had to analyze the whole three-dimensional stack of confocal scans in order to estimate the length of the tubes. Examples are provided by the Movies Ld_1 and Ld_2, which display an Ld vesicle after the first and second deflation step, respectively, and 

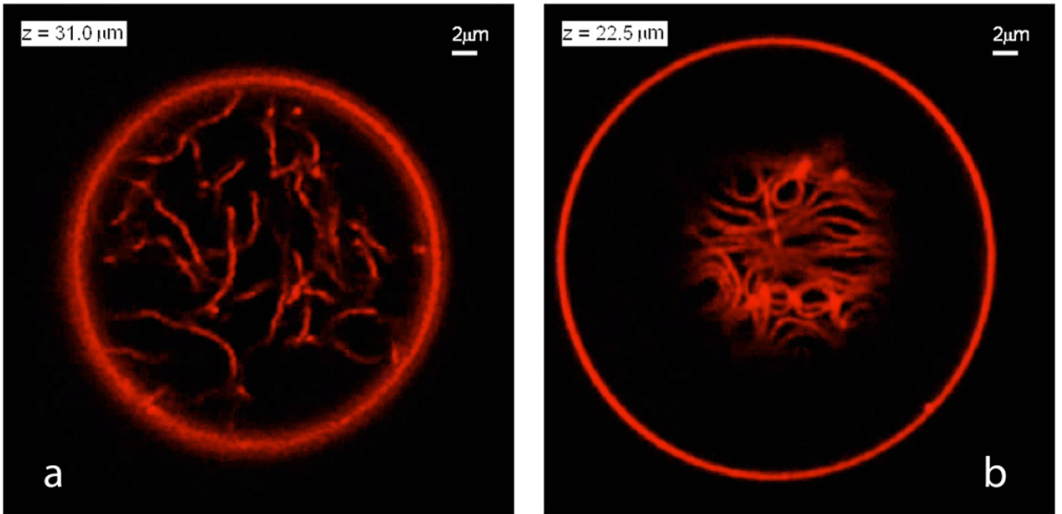

Figure 4. Nanotube patterns within Ld vesicles as observed for the VM-B and VM-C morphologies corresponding to complete and partial wetting of the membranes. (a) Disordered pattern corresponding to an $x y$ scan (with $z=31 \mu \mathrm{m}$ ) of the VM-B morphology after the second deflation step. Because the Ld membrane is completely wetted by the PEG-rich phase, the nanotubes explore the whole PEG-rich droplet but stay away from the dextran-rich phase. (b) Layer of densely packed tubes as visible in an $x y$ scan (with $z=22.5 \mu \mathrm{m}$ ) of the VM-C morphology after the fourth deflation step. As a result of partial wetting, the nanotubes now adhere to the pd interface between the two aqueous droplets and form a thin layer in which crowding leads to short-range orientational order of the tubes. Note that the tube layer is only partially visible because the pd interface is curved into a spherical cap.

reveal that the Ld tubes were quite long, of the order of $20 \mu \mathrm{m}$. For the VM-C morphologies, longer tube segments can be recognized in a single image because the tubes were then localized close to the pd interface between the PEG-rich and the dextran-rich droplet, see Movie Ld_4. This localization is a direct consequence of partial membrane wetting which favors adhesion of the tubes to the pd interface. Thus, the completeto-partial wetting transition can be directly deduced from the observed remodelling of the nanotubes which explore the whole PEG-rich droplet for the VM-B but adhere to the pd interface for the VM-C morphology, see Figure 4.

Because the deflation path was close to the critical consolute point, the tension $\Sigma_{\mathrm{pd}}$ of the pd interface was rather low and varied between 0.1 and $10 \mu \mathrm{N} / \mathrm{m}$, see Figure $3 \mathrm{~b}$. For the VM-C morphology, i.e., for partial wetting of the membranes by the two phases, the pd interface forms a contact line with the membrane and the resulting geometry can be characterized by effective contact angles as defined in Figure S3. The variation of these contact angles with the osmotic deflation is displayed in Figure 3c.

Nucleation and Growth of Necklace-like Tubes. The tubes of the Lo membranes were sufficiently thick to determine their shapes directly from the confocal images. The threedimensional scans in the Movies Lo_1 and Lo_2 show that each Lo vesicle contains many necklace-like tubes consisting of quasi-spherical membrane beads connected by thin membrane necks. The presence of these tubes can be understood from the competition of two kinetic pathways which are related to two different bifurcations of the vesicle shape. Initial deflation of a spherical vesicle leads, via an oblate-stomatocyte bifurcation, ${ }^{26}$ to the formation of a single spherical bud protruding into the vesicle interior, corresponding to the shape $L^{\text {sto }}$ in Figure 5 . Upon further volume reduction, the vesicle can follow two alternative pathways depending on the lipid flow through the narrow membrane neck between the bud and the mother vesicle. If this flow is relatively fast, the bud grows in size until it transforms, via a sphere-to-prolate bifurcation, into a short necklace of two identical spheres, corresponding to the shape $L^{[2]}$ in Figure 5 .

As we continue to deflate the vesicle, the "fast-flow" pathway acts to elongate the necklace-like tube which then passes through the necklace-like shapes $L^{[n]}$ that consist of an increasing number $n$ of small spheres connected by thin membrane necks, see Figure 5. The energy landscape in Figure $5 \mathrm{a}$ and the tube shapes in Figure $5 \mathrm{~b}$ have been obtained by minimizing the bending energy of the membrane, see Methods and Section S1. On the other hand, if the lipid flow through the neck is relatively slow or blocked, the mother vesicle uses the released excess area to form a second bud. Therefore, the deflation of the vesicle generates new buds via the "slow-flow" pathway which are then elongated into necklace-like tubes via the "fast-flow" pathway.

Necklace-Cylinder Coexistence and Critical Tube Length. Even though the vast majority of the Lo tubes were necklace-like, we also observed cylindrical tubes for the VM-C morphology of Lo membranes. Somewhat surprisingly, both necklace-like and cylindrical tubes were found to coexist on the same vesicle. One example is provided by the Lo_4 vesicle displayed in Figure 6 and Movie Lo_4. Close inspection of these confocal images reveals the existence of two long cylindrical tubes that spiral around the spherical cap of the pd interface. These observations can be understood from the competition of different energy contributions which favor necklace-like tubes below a certain critical tube length and cylindrical tubes above this length. At the critical tube length, the necklace-like tube transforms into a cylindrical one. Such a transformation can proceed in a continuous manner via intermediate unduloids as shown in Figure 7.

For the VM-A and VM-B morphologies, the existence of a critical tube length can be understood intuitively from the following simple argument. A necklace-like tube consisting of small spheres with radius $R_{\mathrm{ss}}=1 / \mathrm{Iml}$ as depicted in Figure $7 \mathrm{a}$ has vanishing bending energy. The main body of a cylindrical tube with radius $R_{\mathrm{cy}}=1 /(2|\mathrm{~m}|)$ as displayed in Figure $7 \mathrm{c}$ also has vanishing bending energy, but the cylinder must be closed by two end-caps which have a finite bending energy. Therefore, the bending energy of the membrane disfavors the cylindrical tube. On the other hand, the necklace-like tube has a larger volume compared to the cylindrical one and the osmotic pressure difference across the membranes acts to compress the tubes when they protrude into the interior solution inside the vesicles. $^{8}$ Therefore, such a tube can lower its energy by 


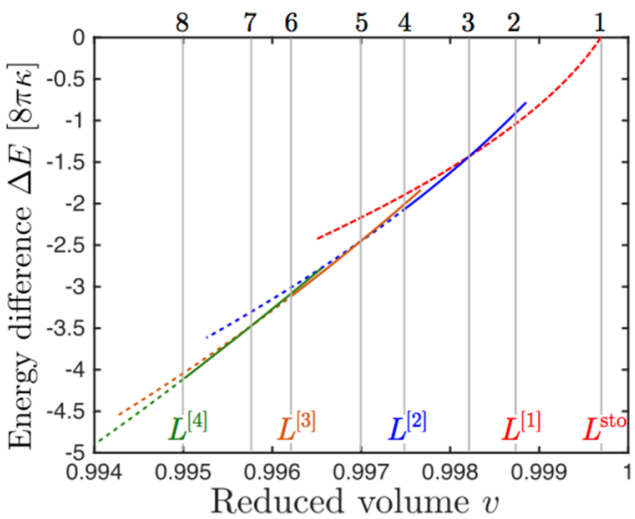

b

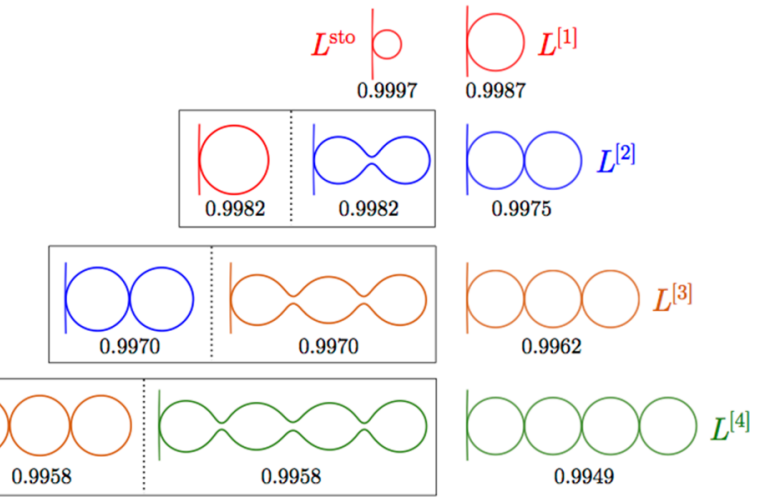

Figure 5. Formation and growth of necklace-like tube. (a) Energy landscape with different branches of equilibrium shapes as a function of the reduced vesicle volume $v$. The latter volume has the value $v=1$ for a spherical shape and continuously decreases during deflation, see Methods. The energy difference $\Delta E$ describes the deflation-induced reduction in bending energy compared to the initial spherical vesicle. The eight vertical lines labeled from 1 to 8 (top) describe eight discrete deflation steps. (b) Shapes of necklace-like tube corresponding to the eight vertical lines in panel a. The short vertical line on the left end of the tubes corresponds to a short segment of the mother vesicle which is connected to each tube by a thin membrane neck. As we deflate the initial vesicle with $v=1$, we move along the 1-necklace branch (red) that begins at the limit shape $L^{\text {sto }}$ with bead radius $R_{\mathrm{ss}} \approx 1 /(2 \mid$ $m \mathrm{l})$ and $v=0.9997$. After passing the shape $L^{[1]}$ with $R_{\mathrm{ss}}=1 / \mathrm{lml}$ and $v=0.9987$, we reach the reduced volume $v=0.9982$ at which the 1 necklace branch crosses the 2-necklace branch (blue). For the latter $v$-value, a 1-necklace coexists with an open 2-necklace. Further deflation leads to the 2-necklace $L^{[2]}$ with a closed neck at $v=$ 0.9975 and, subsequently, to the 3-necklace branch (orange) and the 4-necklace branch (green). The dashed and solid segments of the free energy landscape in panel a correspond to tubes with closed and open necks, respectively. The parameters used in this example belong to the Lo_1 vesicle with size $R_{0}=20.7 \mu \mathrm{m}$ and spontaneous curvature $m=-1.67 \mu \mathrm{m}^{-1}$ as obtained from the area partitioning analysis.

reducing its volume which favors the cylindrical tube. The volume work is proportional to the tube length whereas the bending energy of the end-caps is independent of this length. It then follows from the competition between these two energies that short tubes are necklace-like whereas long tubes are cylindrical.

The same conclusion is obtained from the systematic theory in Section S1 which predicts that an individual necklace-like tube transforms into a cylindrical one when the length of the necklace reaches the critical value $L_{\text {tu }}^{*}$ and that this critical length lies within the interval $2.84 R_{0}<L_{\text {tu }}^{*}<3 R_{0}$ for the observed VM-A and VM-B morphologies where $R_{0} \equiv \sqrt{A_{0} /(4 \pi)}$ represents the radius of the initial vesicle with area $A_{0}$. The upper bound $3 R_{0}$ for the critical tube length is universal and applies to a vesicle with a single tube, whereas the lower bound $2.84 R_{0}$ applies to a vesicle with an arbitrary number of tubes and follows from the observed reduction of the apparent vesicle area during the first two deflation steps. The critical tube area of a single tube is then given by $2 \pi L_{\text {tu }}^{*} /|m|$ which is about $6 \pi R_{0} /|m|$. These bounds for the VM-A and VM$B$ morphologies agree with the observed necklace-like shapes of the Lo tubes which are all shorter than the critical tube length. The Ld tubes are about 5 times longer than the Lo tubes, but they are still shorter than the critical tube length. On the basis of the latter observation and the high flexibility of the Ld tubes, see further below, we conclude that the Ld tubes for the VM-A and VM-B morphologies have a necklace-like shape as well.

For the VM-C morphology, the nanotubes adhere to the pd interface. In this case, the critical tube length is determined by the interplay between (i) the adhesion energy which is proportional to tube length and favors the cylindrical tube and (ii) the bending energy of the end-caps for the cylindrical tube which disfavors the latter tube. The systematic theory in Section S2 now leads to a critical tube length $L_{\text {ad }}^{*}$ that depends on four material parameters, namely the bending rigidity, the spontaneous curvature, the interfacial tension of the $\mathrm{pd}$ interface, and the intrinsic contact angle, as described by eq S47 and Figure S4. All of these parameters have been determined experimentally and two of them are found to vary significantly along the deflation path which implies a corresponding variation of the critical tube length $L_{\text {ad }}^{*}$. The values for the Ld 4 and the Lo 4 vesicle, for example, are given by 5.6 and $21.4 \bar{\mu}$ m, respectively (Figure S4d). The latter value agrees with the length of the cylindrical tubes as observed for the Lo membranes. When we compare the length of individual tubes in Movie Ld 4 with the critical length of $5.6 \mu \mathrm{m}$, we conclude that the L $\overline{\mathrm{d}}$ tubes have a cylindrical shape for this VMC morphology.

Spontaneous Curvature from Image Analysis. For the Lo membranes, the spontaneous curvature can be directly estimated from the shape of the tubes. As mentioned, all Lo tubes found for the VM-A and VM-B morphologies were relatively short and necklace-like which implies that the spontaneous curvature can be estimated via $m=-1 /\left\langle R_{\mathrm{ss}}\right\rangle$ with the average radius $\left\langle R_{\mathrm{ss}}\right\rangle$ of the small, quasi-spherical beads. For the Lo_1 vesicle after the first deflation step, for example, this direct shape analysis leads to $\left\langle R_{\mathrm{ss}}\right\rangle=0.72 \mu \mathrm{m}$ and to the spontaneous curvature $m=-1.4 \mu \mathrm{m}^{-1}$. All $m$-values obtained in this manner are displayed in Figure $8 \mathrm{a}$ as green stars. These values have an accuracy of about $20 \%$, reflecting the relative standard deviation of the measured bead radius $R_{\mathrm{ss}}$.

The direct shape analysis was also applied to the cylindrical and necklace-like tubes coexisting on the Lo_4 vesicle, see Figure 6 . The average diameter $\left\langle 2 R_{\mathrm{cy}}\right\rangle$ of the cylindrical tubes as obtained from the confocal scans (Figure 6d,e) implies the spontaneous curvature $m=-1 /\left\langle 2 R_{\text {cy }}\right\rangle$ which leads to $m=$ $-1.82 \mu \mathrm{m}^{-1}$ for the cylindrical tubes on the Lo_4 vesicle, with an accuracy of about $\pm 13 \%$ corresponding to the relative standard deviation of the measured diameter of the cylinders (Figure 6f). For the latter vesicle, the average bead diameter of the necklace-like tubes leads to $m=-1.56 \mu \mathrm{m}^{-1}$ with an accuracy of about $\pm 19 \%$ (Figure $6 \mathrm{~g}$ ). The good agreement 

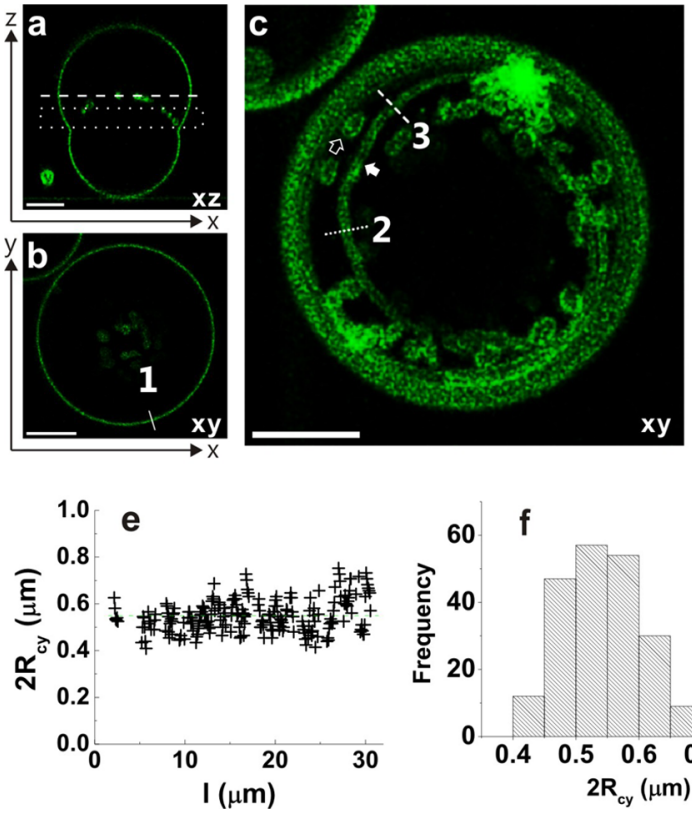
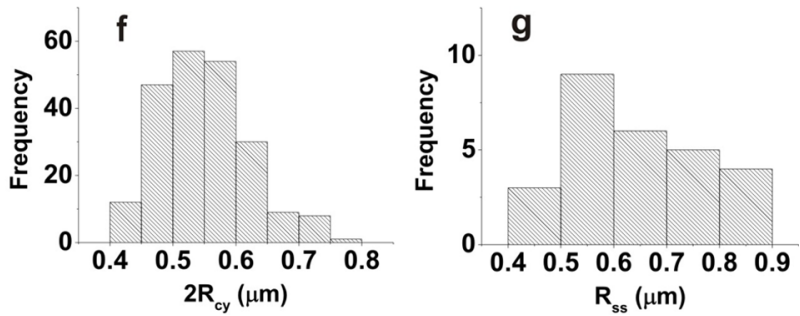

Figure 6. Necklace-cylinder coexistence on Lo_4 vesicle: (a) confocal $x z$-scan; (b) confocal $x y$-scan at $z=29 \mu \mathrm{m}$ corresponding to the dashed line in panel a; (c) superposition of 6 confocal $x y$-scans at $z=20,21,22,23,24$, and $25 \mu \mathrm{m}$ from Movie Lo_4, corresponding to the dotted rectangle in panel a. This superposition reveals the coexistence of two long cylindrical tubes and several short necklace-like tubes, see also Movie Lo_4. All scale bars are $10 \mu \mathrm{m}$. (d) Fluorescent intensity along the solid white line 1 in panel $\mathbf{b}$ perpendicular to the GUV contour and along the dotted and dashed white lines 2 and 3 in panel $c$ across a cylindrical tube. The quantity $\Delta x_{\downarrow}$ is the coordinate perpendicular to the GUV contour or membrane tube. The intensity profiles can be well fitted by Gaussian distributions with a half-peak width of $0.35 \pm 0.05 \mu \mathrm{m}$. The peak-peak separations for the lines 2 and 3 lead to the estimated tube diameters $2 R_{c y}=0.58$ and $0.54 \mu \mathrm{m}$, respectively. (e) The tube diameter $2 R_{\text {cy }}$ as a function of tube position, where $l$ is the distance from the top end of the tube. (f) Cylindrical tubes: histogram of tube diameters $2 R_{\mathrm{cy}}$ with average tube diameter $\left\langle 2 R_{\mathrm{cy}}\right\rangle=0.55 \mu \mathrm{m}$, standard deviation $\sigma_{\mathrm{cy}}=0.07 \mu \mathrm{m}$, and relative standard deviation $\sigma_{\mathrm{cy}} /\left\langle 2 R_{\mathrm{cy}}\right\rangle=$ 0.13. (g) Necklace-like tubes: histogram of bead radii $R_{\mathrm{ss}}$ with average bead radius $\left\langle R_{\mathrm{ss}}\right\rangle=0.64 \mu \mathrm{m}$, standard deviation $\sigma_{\mathrm{ss}}=0.12 \mu \mathrm{m}$, and relative standard deviation $\sigma_{\mathrm{ss}} /\left\langle R_{\mathrm{ss}}\right\rangle=0.19$. Therefore, the direct shape analysis of the cylindrical and necklace-like tubes leads to the estimates $m=-1 /\left(2 R_{\mathrm{cy}}\right)=-1.82 \pm 0.24 \mu \mathrm{m}^{-1}$ and $m=-1 / R_{\mathrm{ss}}=-1.56 \pm 0.30 \mu \mathrm{m}^{-1}$.

a

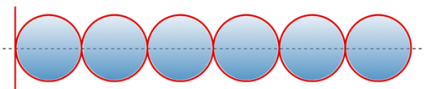

b

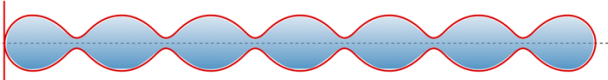

c

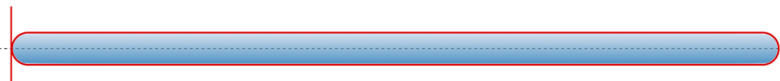

Figure 7. Low energy transformation of a necklace-like tube into a capped cylinder: all three tubes have the same surface area and, apart from the end-caps, the same mean curvature $M$ which is equal to the spontaneous curvature $m$. (a) Necklace-like tube $L^{[6]}$ with vanishing bending energy consisting of six spherical beads connected by thin membrane necks. The small spheres have radius $R_{\mathrm{ss}}=1 / \mid \mathrm{ml}$ and mean curvature $M=-1 / R_{\mathrm{ss}}=m$. (b) Capped unduloid with neck radius $R_{\mathrm{ne}}$, bulge radius $R_{\mathrm{bu}}$ and mean curvature $M=-1 /\left(R_{\mathrm{ne}}+R_{\mathrm{bu}}\right)=m$. (c) Capped cylinder with radius $R_{\text {cy }}=1 /(2|m|)$ and mean curvature $M=-1 /\left(2 R_{\text {cy }}\right)=m$. The transformation of the sphere-necklace into the cylinder proceeds via a continuous family of intermediate unduloids. During this transformation, the tube volume is reduced by a factor 3/4. Apart from their end-caps, the unduloids and the cylinder have the same bending energy as the sphere-necklace. All curvatures are negative because the tubes protrude into the interior aqueous compartments of the vesicles.

between the $m$-values obtained from the cylindrical and necklace-like tubes justifies our implicit assumption that the spontaneous curvature is uniform along the whole membrane of the vesicle.

For the Ld membranes, we could not resolve the shapes of the nanotubes because they had a thickness below optical resolution. However, we could still deduce the spontaneous curvature from two geometric quantities that can be directly determined from the confocal scans: the total tube area $A=A_{0}$ - $A_{\text {app }}$ and the total tube length $L$. The latter length can be directly estimated from the three-dimensional confocal scans, see Movies Ld_j, with an uncertainty of about $\pm 15 \%$. Indeed, all tubes with constant mean curvature are then characterized by spontaneous curvatures within the interval $-2 \pi L / A \leq m \leq$ $-\pi L / A$ (Figure S5). Furthermore, if a fraction $\Lambda$ of the total tube length is cylindrical and the remaining fraction $1-\Lambda$ is necklace-like, we obtain the estimate

$$
m=-(2-\Lambda) \frac{\pi L}{A}=-(2-\Lambda) \frac{\pi L}{A_{0}-A_{\mathrm{app}}}
$$

(area partitioning)

For the VM-A and VM-B morphologies of the Lo membranes, all tubes had a necklace-like shape which implies $\Lambda=0$. After the first deflation step of the Lo vesicle, for example, we measured the excess area $A=354 \mu \mathrm{m}^{2}$ and the overall tube length $L=94 \mu \mathrm{m}$ which leads, via eq 1 with $\Lambda=0$, to the spontaneous curvature $m=-1.67 \mu \mathrm{m}^{-1}$. For the VM-C morphologies, on the other hand, tube adhesion can lead to some cylindrical tubes and nonzero $\Lambda$-values (Table S2). The $m$-values obtained via eq 1 are displayed in Figure $8 \mathrm{a}$ as green 

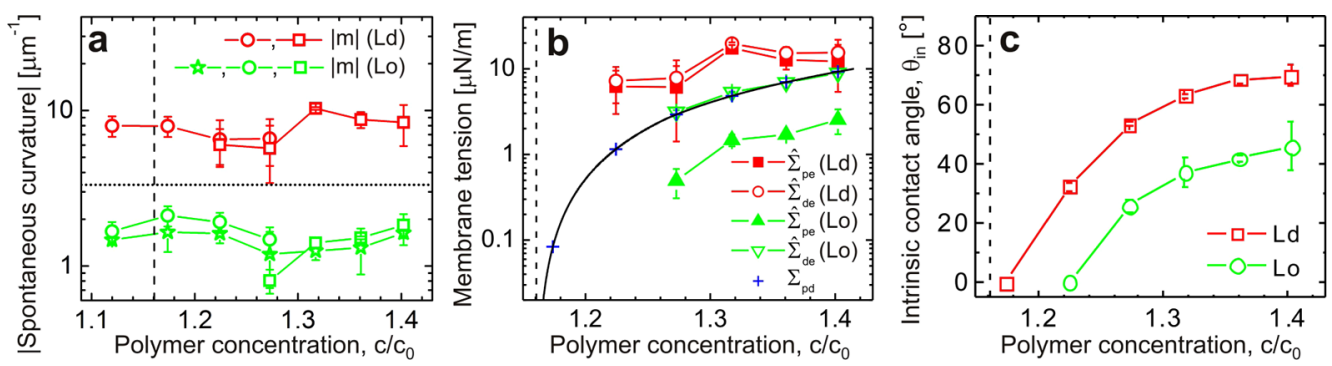

Figure 8. Variation of deduced membrane parameters with osmotic deflation: in all panels, the red and green data correspond to the Ld and Lo membranes, respectively, and the vertical dashed line to the critical concentration $c_{\mathrm{cr}}$. (a) Absolute value of the spontaneous curvature in contact with the uniform aqueous phase (data for $c / c_{0}=1.120$ ) and with the PEG-rich aqueous phase (data for $\left.c / c_{0} \geq 1.174\right)$. The data were obtained by direct shape analysis of the nanotubes (green stars), area partitioning analysis as given by eq 1 (open circles), and force balance analysis described by eq 2 (open squares). The horizontal dotted line corresponds to the optical resolution limit of $1 /(300 \mathrm{~nm})$. (b) Membrane tensions $\hat{\Sigma}_{\mathrm{pe}}$ and $\hat{\boldsymbol{\Sigma}}_{\mathrm{de}}$ within the pe (p, PEG-rich phase; e, exterior phase) and de (d, dextran-rich phase; e, exterior phase) membrane segments separating the external medium from the PEG-rich and dextran-rich phase within the vesicle as defined in Figure S3b. For comparison, the interfacial tension $\boldsymbol{\Sigma}_{\mathrm{pd}}$ is also included (blue crosses and solid black line). (c) Intrinsic contact angle $\theta_{\text {in }}$ between the pd interface and the vesicle membrane as defined in Figure S3c. The intrinsic contact angle is obtained from the effective contact angles via $\cos \left(\theta_{\text {in }}\right)=\left[\sin \left(\theta_{\mathrm{p}}\right)-\sin \left(\theta_{\mathrm{d}}\right)\right] / \sin \left(\theta_{\mathrm{e}}\right) .^{28}$ Note that the intrinsic contact angle, which represents a material parameter, varies rather smoothly with the polymer concentration, in contrast to the effective contact angles in Figure $3 \mathrm{c}$.

open circles and have an accuracy of $\pm 15 \%$, the main uncertainty arising from the measurement of the tube length $L$.

For the Ld tubes, we could not estimate the fraction $\Lambda$ from the confocal scans. However, the high flexibility of these tubes as observed for the VM-A and VM-B morphologies provides strong evidence that these tubes were necklace-like as well. The tube flexibility can be characterized by the persistence length for tube bending. Using the parameter values for the $\mathrm{Ld}$ membranes, we then find that a cylindrical tube has a persistence length of $15 \mu \mathrm{m}$ (Section S3), whereas the persistence length of the corresponding necklace-like tube should be comparable to the diameter of the small spheres. Inspection of the Movies Ld_1 and Ld_2 reveals that the thermal fluctuations of the $\overline{L d}$ tubes lead to hairpin-like conformations with curvature radii of the order of $2 \mu \mathrm{m}$ which implies a persistence length below $2 \mu \mathrm{m}$. Such an upper bound for the persistence length is consistent with a necklace-like but not with a cylindrical tube morphology. As mentioned, the same conclusion is obtained from the observed tube lengths which are shorter than the critical tube lengths for the necklacecylinder transformation. Therefore, we estimated the spontaneous curvatures for the VM-A and VM-B morphologies of the Ld membranes using eq 1 with $\Lambda=0$. The spontaneous curvature of the Ld_1 vesicle, for example, is then found to be $m=-1 /(125 \mathrm{~nm})$. For the VM-C morphologies of the $\mathrm{Ld}$ membranes, we deduced the total tube length $L$ from the density of the tubes at the pd interface and estimated the spontaneous curvature via eq 1 using the value $\Lambda=\frac{1}{2} \pm \frac{1}{2}$, thereby taking the whole range $0 \leq \Lambda \leq 1$ of possible $\Lambda$-values into account.

For the VM-C morphologies of both the Lo and the Ld membranes, the values of the spontaneous curvature as deduced from the direct shape and the area partitioning analysis could be validated via a third, completely different mode of analysis. The latter mode is based on the interfacial tension $\Sigma_{\mathrm{pd}}$ of the pd interface (Figure $3 \mathrm{~b}$ ) as well as on the effective contact angles (Figure 3c). ${ }^{7}$ Using these quantities and the force balance along the contact line, we computed the membrane tensions in the two segments (Figure $8 \mathrm{~b}$ ) and the spontaneous curvature via

$$
m=-\left(\frac{\Sigma_{\mathrm{pd}}}{2 \kappa} \frac{\sin \left(\theta_{\mathrm{d}}\right)}{\sin \left(\theta_{\mathrm{e}}\right)}\right)^{1 / 2} \quad \text { (force balance) }
$$

With the use of the measured bending rigidities for the $\mathrm{Ld}$ and Lo membranes, the expression given by eq 2 leads to $m$-values that are in good agreement with those obtained via the two other modes of analysis, see open squares in Figure 8a.

Spontaneous Curvature Generated by PEG Adsorption. Because vesicle tubulation was only observed in the presence of the polymers, the spontaneous curvature of the vesicle membranes must arise from the polymer-membrane interactions. If these interactions are effectively attractive or repulsive, the polymers form adsorption or depletion layers on the two bilayer leaflets. The membrane then prefers to bulge toward the solutions with the higher and lower concentrations for polymer adsorption and depletion, respectively. ${ }^{29}$ After the first deflation step, both the PEG and the dextran concentrations in the interior aqueous solution are larger than in the exterior solution (Figure 2a). After the second and all subsequent deflation steps, the PEG concentration in the interior PEG-rich phase is again larger than in the exterior solution, but the dextran concentration in the interior phase is now smaller than in this exterior solution (Figure 2a). Furthermore, all deflation steps lead to a negative spontaneous curvature of the membranes. These observations are only consistent with the theoretical results in ref 29 if the spontaneous curvature is induced by PEG adsorption. The latter conclusion has been confirmed by additional experiments in which we used the same lipid composition but exposed the vesicles to aqueous solutions that contained only PEG and no dextran, see Figure 9. For the latter systems, osmotic deflation of the vesicles again generated membrane nanotubes that protruded into the solution with the higher PEG concentration.

To corroborate these conclusions about PEG adsorption and to obtain direct insight into the conformations of the adsorbed PEG molecules, we performed molecular dynamics simulations of the polymer-lipid systems with atomistic resolution for the experimentally used lipid compositions and chain length of PEG, see Methods. Typical conformations of adsorbed chains are displayed in Figure 10a,b for both Ld and Lo membranes. These conformations indicate that the PEG molecules are only 

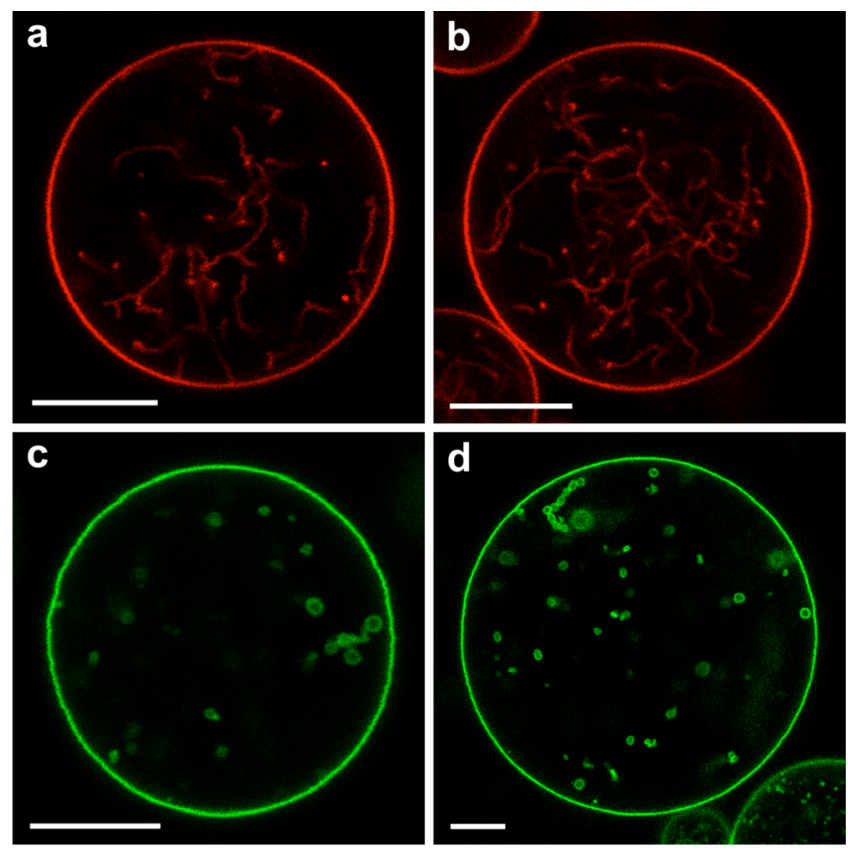

Figure 9. Nanotubes formed in Ld (red) and Lo (green) vesicles exposed to aqueous solutions of PEG and sucrose, i.e., in the absence of dextran. The interior solution contained only PEG and no sucrose with the initial weight fraction $w_{\mathrm{p}}=0.0443$. The vesicles were deflated by exchanging the external medium by a hypertonic solution with no PEG but an increasing weight fraction $w_{\text {su }}$ of sucrose. The vesicles in (a) and (c) are obtained for $\boldsymbol{w}_{\mathrm{su}}=\mathbf{0 . 0 0 6 6}$, those in (b) and (d) for $w_{\mathrm{su}}=\mathbf{0 . 0 1}$. The corresponding osmolarity ratios $P_{\mathrm{e}} / P_{0}$ are 1.05 and 1.61 , respectively. The white scale bars are $10 \mu \mathrm{m}$ in all panels.

weakly bound, with relatively short contact segments (or "trains"), and relatively long loops in between. The two terminal $\mathrm{OH}$ groups of the PEG molecule were frequently bound to the membrane via hydrogen bonds. In addition, a small number of contacts was formed between the polymer backbones and the membranes. Combining both types of contacts, the adsorbed polymers formed an average number of $4.5 \pm 2.3$ and $3.2 \pm 2.1$ contacts with the $\mathrm{Ld}$ and the Lo membranes, respectively. A more quantitative measure for the affinity of the polymers to the membranes is provided by the potentials of mean force displayed in Figure 10c,d. These potentials indicate that the PEG molecules have essentially the same affinity for both types of membranes, with a binding free energy of about $4 \mathrm{~kJ} / \mathrm{mol}$ or $1.6 k_{\mathrm{B}} T$ per polymer chain.

Discussion of Deduced Spontaneous Curvatures. The spontaneous curvatures displayed in Figure $8 \mathrm{a}$ have two remarkable features: (i) they depend only weakly on the total polymer concentration $c$ within the vesicles; and (ii) when exposed to the same asymmetric environment, the spontaneous curvature of the Ld membrane is about 4.7 times larger than that of the Lo membrane. The second feature is relatively easy to explain. Indeed, the spontaneous curvature generated by adsorption is theoretically predicted to be inversely proportional to the bending rigidity of the membrane ${ }^{8,29}$ as recently confirmed for the adsorption of small molecules by molecular dynamics simulations. ${ }^{30}$ If we assume that the PEG chains have similar affinities to the Ld and Lo membranes as implied by our simulation results (Figure $10 \mathrm{c}, \mathrm{d}$ ), the ratio $m_{\mathrm{Ld}} / m_{\mathrm{Lo}}$ of the spontaneous curvatures should be equal to the ratio $\kappa_{\mathrm{Lo}} / \kappa_{\mathrm{Ld}}$ of the bending rigidities. The latter rigidity ratio is estimated to be
$\kappa_{\mathrm{Lo}} / \kappa_{\mathrm{Ld}} \simeq 4.5$, based on the experimental results in ref 25 , which should be compared with the curvature ratio $m_{\mathrm{Ld}} / m_{\mathrm{Lo}} \simeq 4.7 \mathrm{in}$ Figure $8 \mathrm{a}$. Because the experimental uncertainty is of the order of 10 to $20 \%$ for both ratios, the data are consistent with $m_{\mathrm{Ld}} /$ $m_{\mathrm{Lo}} \simeq \kappa_{\mathrm{Lo}} / \kappa_{\mathrm{Ld}}$ and, thus, with a comparable amount of adsorbed polymers on both types of membranes, in agreement with our simulation results.

The weak dependence of the spontaneous curvatures on the polymer concentration $c$ within the vesicles is more difficult to understand. Because the polymer weight fractions in the exterior solution were kept constant during all deflation steps, the concentration-dependence of the spontaneous curvatures follows from the concentration-dependence of the PEG coverage on the inner membrane leaflets in contact with the interior polymer solutions. If these leaflets were in contact with dilute PEG solutions, the polymer density within the adsorption layer would be increased by a factor of about $\exp \left[\Delta F_{\mathrm{ch}} / k_{\mathrm{B}} T\right] \simeq$ 5 compared to the bulk density, based on the binding free energy $\Delta F_{\mathrm{ch}} \simeq 1.6 k_{\mathrm{B}} T$ for a single PEG chain (Figure 10c,d). Therefore, as the polymer concentration within the interior solution is increased, the PEG coverage on the interior leaflets of the membranes would also be increased which implied a corresponding increase of the spontaneous curvature.

However, for the deflation path studied here, the PEGdextran solutions were not dilute but semidilute, with substantial overlaps between the chains, see Table S3, which led to repulsive PEG-dextran interactions in the one-phase region and to repulsive PEG-PEG interactions in the PEG-rich phase. When the PEG chains are adsorbed onto the inner leaflet, the local PEG concentration is increased and the PEG chains must experience even more repulsive PEG-PEG and PEG-dextran interactions than in the bulk solutions. For adsorption onto solid surfaces, repulsive chain-chain interactions typically lead to a saturation of the polymer coverage as observed in many experiments, see, e.g., refs $31-33$. In the present context, such a saturation of the PEG coverage is quite plausible after the third deflation step. Furthermore, after the first and the second deflation step, the interior polymer solutions were rather close to the critical consolute point with $\epsilon$ $\equiv \mathrm{I}_{c}-c_{\mathrm{cr}} \mathrm{I} / \mathcal{c}_{\mathrm{cr}}$ of the order of $10^{-2}$ (Table S3). In such a situation, the correlation length for compositional fluctuations is expected to be large compared to the size of the PEG chains which implies a thickening of the adsorption layers and a corresponding increase in the PEG coverage on the inner membrane leaflets. Thus, the weak concentration-dependence of this coverage should arise from the antagonistic effects of near-critical fluctuations and repulsive chain-chain interactions along the chosen deflation path.

\section{CONCLUSIONS}

In summary, we have shown that both Ld and Lo membranes form nanotubes when their leaflets are exposed to two aqueous polymer solutions that differ in their composition. The total length of the tubes is controlled by the osmotic deflation, while their thickness directly reflects the bilayer asymmetry of the membranes as described by their spontaneous curvature. The vast majority of individual Lo nanotubes had a necklace-like shape (Figure 2 as well as Figure S2 and Movies), but we also observed long cylindrical tubes coexisting with shorter necklace-like tubes on the same vesicle (Figure 6 and Movie Lo 4). The formation of many necklace-like tubes can be understood from the competition of two kinetic pathways. The first pathway nucleates new buds of the mother vesicle whereas 
a

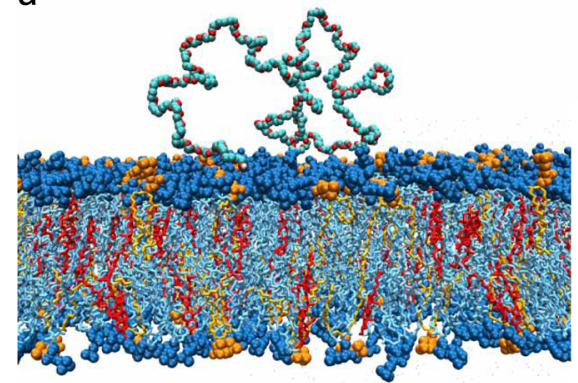

Ld membrane

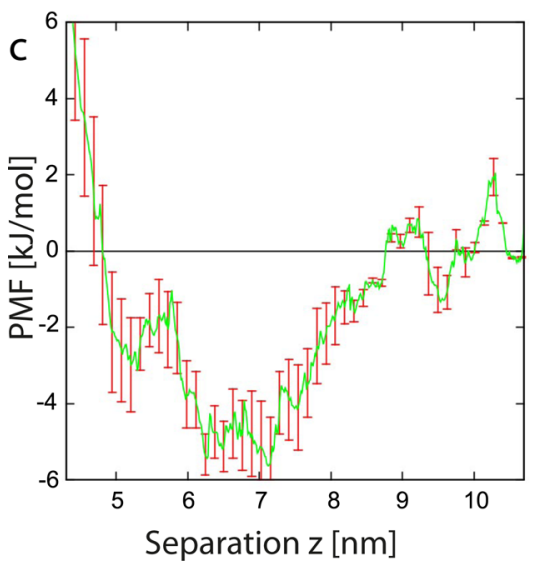

b

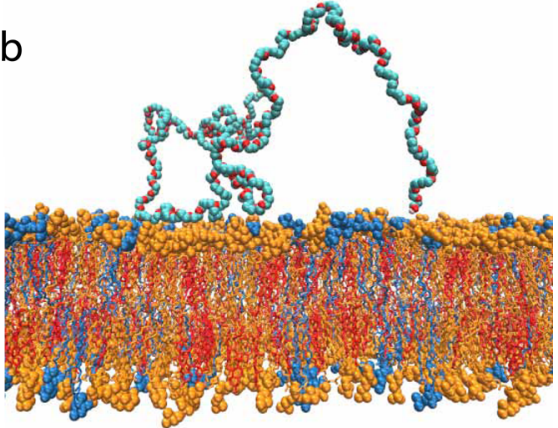

Lo membrane

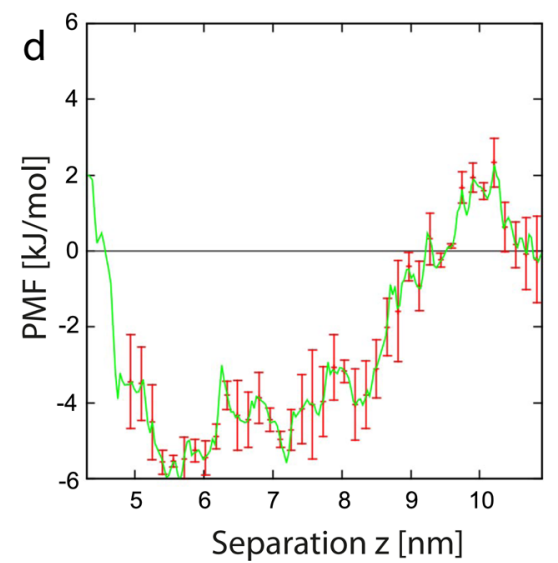

Figure 10. Typical conformation and potential of mean force for adsorbed PEG molecules. (a and b) Simulation snapshots of PEG molecule adsorbed onto Ld and Lo bilayer. The Ld and Lo composition is the same as in Figure 2. The color code for the lipids is blue for DOPC, orange for DPPC, and red for cholesterol. The PEG molecules consist of 180 monomers corresponding to the average molecular weight used in the experiments. Each lipid membrane is immersed in about 27000 water molecules (not shown). (c and d) Potential of mean force (PMF) for Ld and Lo membranes as a function of the separation $z$ between the polymer's center-of-mass and the bilayer's midplane. The potential wells are relatively broad, with a width of about $4 \mathrm{~nm}$, because the polymer end groups can adsorb even for relatively large $z$-values. The binding free energy of a single PEG chain is about $4 \mathrm{~kJ} / \mathrm{mol}$ or $1.6 k_{\mathrm{B}} T$ for both types of membranes.

the second pathway stores additional area in the existing buds and extends these buds into necklace-like tubes (Figure $5 b$ ). These necklace-like tubes transform into cylindrical tubes when their length reaches a certain critical length (Sections S1 and S2). It then follows from the magnitude of the critical tube lengths that individual Ld tubes were necklace-like for the VMA and VM-B morphologies but cylindrical for some of the VM$\mathrm{C}$ morphologies. In the latter case, the tube adhesion led to a reduction of the critical tube length.

To deduce the spontaneous curvature from the observed vesicle morphologies, we used three different and independent methods of image analysis: direct shape analysis (Figure 6), overall partitioning analysis of membrane area (eq 1), and force balance analysis along the contact line of the partially wetted membranes (eq 2). All three analysis methods gave very consistent values for the spontaneous curvature $m$ as summarized in Figure 8a. The spontaneous curvatures of the Ld and Lo membranes were fairly constant over the range of polymer concentrations studied here, with $m_{\mathrm{Ld}} \simeq-8 \mu \mathrm{m}^{-1}$ and $m_{\mathrm{Lo}} \simeq-1.7 \mu \mathrm{m}^{-1}$ which implies the curvature ratio $m_{\mathrm{Ld}} / m_{\mathrm{Lo}} \simeq$ 4.7.

We also elucidated the molecular mechanism for the generation of the spontaneous curvatures and showed that these curvatures are induced by the weak adsorption of PEG molecules onto the membranes. Indeed, the formation of nanotubes was also observed in the absence of dextran, e.g., when the giant vesicles were exposed to aqueous solutions of
PEG alone (Figure 9). In addition, both the typical conformations of the adsorbed polymers and the associated polymer-membrane affinities were determined by atomistic molecular dynamics simulations (Figure 10). The binding free energies were found to be relatively small and similar in size for Ld and Lo membranes, about $4 \mathrm{~kJ} / \mathrm{mol}$ or $1.6 k_{\mathrm{B}} T$ per polymer chain. Because of this similarity, the curvature ratio $m_{\mathrm{Ld}} / m_{\mathrm{Lo}}$ is approximately equal to the rigidity ratio $\kappa_{\mathrm{Lo}} / \kappa_{\mathrm{Ld}}$.

Our study can be extended in several ways. First, it will be useful to further corroborate our preliminary results for dilute PEG solutions (Figure 9) in order to avoid the added complexity of semidilute polymer mixtures. Second, using other lipid or polymer compositions with different bending rigidities or polymer-membrane affinities, we can easily vary the tube thickness and, in particular, produce thinner and longer nanotubes which should then exhibit a larger fraction of cylinders. Third, using osmotic deflation in combination with micropipette aspiration to shorten the tubes ${ }^{7}$ and/or optical tweezers to extend individual tubes, one should be able to directly probe the dynamics of the necklace-cylinder transformation.

Because our deflation-based approach is both simple and general, it can be applied to other membrane systems of interest. One example is provided by synthetic lipid bilayers with two leaflets that have different compositions as recently produced by a variety of preparation methods. ${ }^{34-38}$ With the use of similar methods, it should also be possible to produce 
bilayers of block copolymers with such a compositional asymmetry. Block copolymer bilayers form giant polymersomes $^{14}$ which change their shapes in response to osmotic deflation $^{15,16,39}$ and can form tubular shapes as well. ${ }^{11}$ Furthermore, our approach can also be used to determine the spontaneous curvature arising from the membrane binding of BAR-domain proteins ${ }^{17,18}$ and amphipathic peptides. ${ }^{19}$

In the living cell, the membranes are continuously remodelled by the formation of transport vesicles via fission and fusion processes. The magnitude of the spontaneous curvature determines whether these remodelling processes are endergonic or exergonic. If the spontaneous curvature is comparable to the inverse size of the transport vesicle, budding is exergonic and fusion is endergonic. One example for the latter situation is provided by clathrin-dependent endocytosis for which the spontaneous curvature plays a pivotal role. ${ }^{20}$

\section{METHODS}

Materials. Dextran $(400-500 \mathrm{~kg} / \mathrm{mol})$ and poly(ethylene glycol) (PEG 8000, $8 \mathrm{~kg} / \mathrm{mol}$ ) were purchased from Sigma-Aldrich. The polydispersity, measured with gel permeation chromatography, was 1.11 for PEG and 1.83 for dextran. The binodal of the polymer solution was determined by cloud-point titration. ${ }^{22}$ The critical consolute (or demixing) point was located at a total polymer concentration of 8.12 wt $\%$ with a dextran to PEG weight ratio $w_{\mathrm{d}} / w_{\mathrm{p}}$ $=1.25$. The interfacial tensions $\Sigma_{\mathrm{pd}}$ between the dextran-rich and PEGrich phases were measured using a SITE100 spinning drop tensiometer (Krüss) as described in ref 22. Dioleoylphosphatidylcholine (DOPC) and dipalmitoylphosphatidylcholine (DPPC) were purchased from Avanti Polar Lipids and cholesterol from SigmaAldrich. Texas Red labeled dihexadecanoyl phosphoethanolamine (DHPE-TR) was purchased from Invitrogen and distearoyl phosphoethanolamine- $N$-[poly(ethylene glycol)2000- $N^{\prime}$-carboxyfluorescein] (DSPE-PEG-CF) from Avanti Polar Lipids.

Vesicle Preparation and Deflation. Aqueous polymer solutions with initial weight fraction $\left(w_{\mathrm{d}}, w_{\mathrm{p}}\right)_{0}=(0.0390,0.0312)$ corresponding to composition 0 (blue cross in Figure $2 \mathrm{a}, \mathrm{b}$ ) were encapsulated within giant vesicles with membranes composed of DOPC, DPPC and cholesterol. The latter vesicles were formed using the electroformation method as described elsewhere. ${ }^{7}$ The Ld membranes with lipid composition DOPC:DPPC: cholesterol = 64:15:21 were labeled by 0.1 mol \% DHPE-TR. The Lo membranes with lipid composition DOPC:DPPC:cholesterol $=13: 44: 43$ were labeled with $0.2 \mathrm{~mol} \%$ DSPE-PEG-CF. For these two lipid compositions, the bending rigidities were previously studied; we used the values $\kappa_{\mathrm{Ld}}=0.82 \times$ $10^{-19} \mathrm{~J}$ and $\kappa_{\mathrm{Lo}}=3.69 \times 10^{-19} \mathrm{~J}$ obtained in ref 25 . After preparation, the vesicles were transferred into a chamber filled with an isotonic solution with polymer weight fractions $\left(w_{\mathrm{d}}, w_{\mathrm{p}}\right)_{0 e}=(0.0327,0.0327)$, corresponding to the green cross in Figure 2a,b. The deflation was done by exchanging the external medium with a hypertonic solution containing the constant polymer weight fractions $w_{\mathrm{d}}=0.0327$ and $w_{\mathrm{p}}=$ 0.0327 and an increasing weight fraction of sucrose. These conditions ensured that the vesicles sedimented toward the coverslip at the bottom of the chamber and that the symmetry axis was oriented perpendicular to this coverslip; see Figure 2c,d and Figure S2a,b.

Confocal Microscopy. Vesicles were observed by a confocal microscope (Leica TCS SP5) with a $63 \times$ water immersion objective $($ N.A. $=1.20)$. The images were deconvoluted by Huygens Professional software (version 4.3.1, Scientic Volume Imaging), and analyzed using home-developed software in Matlab to determine the contours of the mother vesicle and the membrane nanotubes. A complete three-dimensional scan of a vesicle consisted of a stack of 37-82 confocal images that were taken at different separations from the coverslip, see the Movies Ld $\_j$ and Lo $j$ which display scans of Ld and Lo vesicles after the first, second, and fourth depletion step. The analysis of these scans is described in more detail in Figure 6 and Table S2.
Computation of Vesicle Shapes via Free Energy Minimization. For the VM-A and VM-B morphologies, the free energy landscapes of the giant vesicles have been determined by minimizing the bending energy of the vesicle membrane for constant membrane area $A_{0}$ and deflation-controlled vesicle volume $V$. The bending energy depends (i) on the shape of the vesicle membrane which is described by its (local) mean curvature $M$ and (ii) on two fluid-elastic parameters, the bending rigidity $\kappa$ and the spontaneous curvature $m$. ${ }^{26,40}$ The constraints on area and volume are taken into account by two Lagrange multipliers, $\Sigma$ and $\Delta P$, and by minimizing the shape functional ${ }^{26}$

$$
\mathcal{E}=\int \mathrm{d} A 2 \kappa(\mathrm{M}-\mathrm{m})^{2}+\Sigma A_{0}-\Delta P V
$$

We used the vesicle size $R_{0} \equiv\left(A_{0} / 4 \pi\right)^{1 / 2}$ as the basic length scale and the volume-to-area ratio or reduced volume

$$
v \equiv V /\left[(4 \pi / 3) R_{0}^{3}\right] \leq 1
$$

as the basic control parameter or reaction coordinate, see Sect. S1 for more details. In this way, we obtained the free energy landscape for a giant vesicle with a necklace-like tube as displayed in Figure 5.

For the VM-C morphology, the overall shape of the vesicles can be determined by an appropriate generalization of the shape functional as described in ref 28. The VM-C shape involves the intrinsic contact angle $\theta_{\text {in }}$ as an additional material parameter, see Figure S3c. In order to study the adhesion of membrane nanotubes to the pd interface, we ignored the relatively small curvature of the pd interface and calculated the free energies for the adhesion of both necklace-like and cylindrical tubes to a planar pd interface. The results of these calculations are described in Section S2 and summarized in Figure S4.

Molecular Dynamics Simulations of PEG Adsorption. For the atomistic simulations, we used the GROMACS software package 4.5.5 with the Berger force field ${ }^{41}$ for the lipids and parameters based on the GROMOS 53A6 force field for the polymers. ${ }^{42}$ For the PEG 8000 polymer chain, this force field leads to a radius of gyration of $3.43 \mathrm{~nm}$ in reasonable agreement with the value $3.83 \mathrm{~nm}$ for PEG 8000 as obtained by linear extrapolation from the results in ref 43 and the value $4.05 \mathrm{~nm}$ as estimated in ref 22 based on the PEO data in ref 44. Lipid bilayers corresponding to the experimentally used compositions for the Ld and Lo membranes were assembled, together with a single polymer chain of PEG 8000 , in a cubic simulation box of size $10.3 \times$ $10.3 \times 14.8 \mathrm{~nm}^{3}$ with periodic boundary conditions in all three spatial directions for the unrestrained simulations, using the charmm membrane builder. ${ }^{45}$ The Ld membrane contained 256 DOPC, 60 DPPC, and 84 cholesterol molecules, whereas the Lo membrane consisted of 52 DOPC, 176 DPPC, and 172 cholesterol molecules (Figure 10). The PEG 8000 molecules had a length of 180 monomers. Bilayer and polymer were immersed in 27080 water molecules as described by the SPC water model. Both the Ld and the Lo membranes were simulated for 700 ns. For the computations of the potentials of mean force in Figure 10c,d, we increased the box size perpendicular to the membrane to $20.5 \mathrm{~nm}$ corresponding to 51758 water molecules and used 12 umbrella windows, each of which was simulated for $100 \mathrm{~ns}$.

\section{ASSOCIATED CONTENT}

\section{Supporting Information}

The Supporting Information is available free of charge on the ACS Publications website at DOI: 10.1021/acsnano.5b05377.
Additional experimenta
Movie Ld 1 (MPG)
Movie Ld 2 (MPG)
Movie Ld 4 (MPG)
Movie Lo_1 (MPG)
Movie Lo_2 (MPG)
Movie Lo_4 (MPG) 


\section{AUTHOR INFORMATION}

\section{Corresponding Author}

*E-mail: lipowsky@mpikg.mpg.de.

\section{Notes}

The authors declare no competing financial interest.

\section{ACKNOWLEDGMENTS}

We thank Halim Kusumaatmaja for stimulating discussions during the initial phase of this project and acknowledge support by the Partner Group Program of the Max Planck Society and the Chinese Academy of Sciences, the National Natural Science Foundation of China (Grant No. 21274147), the German Science Foundation (DFG) via IRTG 1524, and the Federal Ministry of Education and Research (BMBF) via the consortium MaxSynBio.

\section{REFERENCES}

(1) Marchi, S.; Patergnani, S.; Pinton, P. The Endoplasmic Reticulum-Mitochondria Connection: One Touch, Multiple Functions. Biochim. Biophys. Acta, Bioenerg. 2014, 1837, 461-469.

(2) van Weering, J. R. T.; Cullen, P. J. Membrane-Associated Cargo Recycling by Tubule-Based Endosomal Sorting. Semin. Cell Dev. Biol. 2014, 31, 40-47.

(3) Westrate, L. M.; Lee, J. E.; Prinz, W. A.; Voeltz, G. K. Form Follows Function: The Importance of Endoplasmic Reticulum Shape. Annu. Rev. Biochem. 2015, 84, 791-811.

(4) Wang, X.; Gerdes, H.-H. Transfer of Mitochondria via Tunneling Nnotubes Rescues Apoptotic PC12 Cells. Cell Death Differ. 2015, 22, $1181-1191$

(5) He, K.; Luo, W.; Zhang, Y.; Liu, F.; Liu, D.; Xu, L.; Qin, L.; Xiong, C.; Lu, Z.; Fang, X.; Zhang, Y. Intercellular Transportation of Quantum Dots Mediated by Membrane Nanotubes. ACS Nano 2010, 4, 3015-3022.

(6) Sowinski, S.; Jolly, C.; Berninghausen, O.; Purbhoo, M. A.; Chauveau, A.; Köhler, K.; Oddos, S.; Eissmann, P.; Brodsky, F. M.; Hopkins, C.; et al. Membrane Nanotubes Physically Connect T Cells over Long Distances Presenting a Novel Route for HIV-1 Transmission. Nat. Cell Biol. 2008, 10, 211-219.

(7) Li, Y.; Lipowsky, R.; Dimova, R. Membrane Nanotubes Induced by Aqueous Phase Separation and Stabilized by Spontaneous Curvature. Proc. Natl. Acad. Sci. U. S. A. 2011, 108, 4731-4736.

(8) Lipowsky, R. Spontaneous Tubulation of Membranes and Vesicles Reveals Membrane Tension Generated by Spontaneous Curvature. Faraday Discuss. 2013, 161, 305-331.

(9) Sorre, B.; Callan-Jones, A.; Manzi, J.; Goud, B.; Prost, J.; Bassereau, P.; Roux, A. Nature of Curvature Coupling of Amphiphysin with Membranes Depends on its Bound Density. Proc. Natl. Acad. Sci. U. S. A. 2012, 109, 173-178.

(10) Zhu, C.; Das, S. L.; Baumgart, T. Nonlinear Sorting, Curvature Generation, and Crowding of Endophilin N-Bar on Tubular Membranes. Biophys. J. 2012, 102, 1837-1845.

(11) Robertson, J. D.; Yealland, G.; Avila-Olias, M.; Chierico, L.; Bandmann, O.; Renshaw, S. A.; Battaglia, G. pH-Sensitive Tubular Polymersomes: Formation and Applications in Cellular Delivery. ACS Nano 2014, 8, 4650-4661.

(12) Tanaka, M.; Critchley, K.; Matsunaga, T.; Evans, S. D.; Staniland, S. S. Fabrication of Lipid Tubules with Embedded Quantum Dots by Membrane Tubulation Protein. Small 2012, 8, 1590-1595.

(13) Albertsson, P. A. Partition of Cell Particles and Macromolecules: Separation and Purification of Biomolecules, Cell Organelles, Membranes, and Cells in Aqueous Polymer Two-Phase Systems and Their Use in Biochemical Analysis and Biotechnology, 3rd ed.; Wiley, 1986.

(14) Discher, B. M.; Won, Y.-Y.; Ege, D. S.; Lee, J. C.-M.; Bates, F. S.; Discher, D. E.; Hammer, D. A. Polymersoms: Tough Vesicles Made from Diblock Copolymers. Science 1999, 284, 1143-1146.
(15) Salva, R.; Meins, J.-F. L.; Sandre, O.; Brulet, A.; Schmutz, M.; Guenoun, P.; Lecommandoux, S. Polymersome Shape Transformation at the Nanoscale. ACS Nano 2013, 7, 9298-9311.

(16) Thiele, J.; Chokkalingam, V.; Ma, S.; Wilson, D. A.; Huck, W. T. S. Vesicle Budding from Polymersomes Templated by Microfuidically Prepared Double Emulsions. Mater. Horiz. 2014, 1, 96-101.

(17) McMahon, H. T.; Gallop, J. L. Membrane Curvature and Mechanisms of Dynamic Cell Membrane Remodelling. Nature 2005, 438, 590-596.

(18) Rao, Y.; Haucke, V. Membrane Shaping by the Bin/ Amphitaphysin/Rvs (BAR) Domain Protein Superfamily. Cell. Mol. Life Sci. 2011, 68, 3983-3993.

(19) Arouni, A.; Kiessling, V.; Tamm, L.; Dathe, M.; Blume, A. Morphological Changes Induced by the Action of Antimicrobial Peptides on Supported Lipid Bilayers. J. Phys. Chem. B 2011, 115, $158-167$.

(20) Agudo-Canalejo, J.; Lipowsky, R. Critical Particle Sizes for the Engulfment of Nanoparticles by Membranes and Vesicles with Bilayer Asymmetry. ACS Nano 2015, 9, 3704-3720.

(21) Helfrich, M.; Mangeney-Slavin, L.; Long, M.; Djoko, K.; Keating, C. Aqueous Phase Separation in Giant Vesicles. J. Am. Chem. Soc. 2002, 124, 13374-13375.

(22) Liu, Y.; Lipowsky, R; Dimova, R. Concentration Dependence of the Interfacial Tension for Aqueous Two-Phase Polymer Solutions of Dextran and Polyethylene Glycol. Langmuir 2012, 28, 3831-3839.

(23) Veatch, S.; Keller, S. Separation of Liquid Phases in Giant Vesicles of Ternary Mixtures of Phospholipids and Cholesterol. Biophys. J. 2003, 85, 3074-3083.

(24) Tian, A.; Capraro, B. R.; Esposito, C.; Baumgart, T. Bending Stiffness Depends on Curvature of Ternary Lipid Mixture Tubular Membranes. Biophys. J. 2009, 97, 1636-1646.

(25) Heinrich, M.; Tian, A.; Esposito, C.; Baumgart, T. Dynamic Sorting of Lipids and Proteins in Membrane Tubes with a Moving Phase Boundary. Proc. Natl. Acad. Sci. U. S. A. 2010, 107, 7208-7213.

(26) Seifert, U.; Berndl, K.; Lipowsky, R. Shape Transformations of Vesicles: Phase Diagram for Spontaneous Curvature and Bilayer Coupling Model. Phys. Rev. A: At., Mol., Opt. Phys. 1991, 44, 11821202.

(27) Lipowsky, R. The Conformation of Membranes. Nature 1991, $349,475-481$.

(28) Kusumaatmaja, H.; Li, Y.; Dimova, R.; Lipowsky, R. Intrinsic Contact Angle of Aqueous Phases at Membranes and Vesicles. Phys. Rev. Lett. 2009, 103, 238103.

(29) Breidenich, M.; Netz, R.; Lipowsky, R. The Influence of NonAnchored Polymers on the Curvature of Vesicles. Mol. Phys. 2005, 103, 3169-3183.

(30) Rózycki, B.; Lipowsky, R. Spontaneous Curvature of Bilayer Membranes from Molecular Simulations: Asymmetric Lipid Densities and Asymmetric Adsorption. J. Chem. Phys. 2015, 142, 054101.

(31) Felter, R. E.; Ray, L. N. Polymer Adsorption Studies at the Solid-Liquid Interface Using Gel Permeation Chromatography. I. Colloid Interface Sci. 1970, 32, 349-360.

(32) Voronov, A.; Luzinov, I.; Minko, S.; Sidorenko, A. Adsorption of Polymers at the Surface Concentrations in the Diluted to Semidiluted Regimes. Macromolecules 1997, 30, 6929-6936.

(33) Liufu, S.; Xiao, H.; Li, Y. Investigation of PEG Adsorption on the Surface of Zinc Oxide Nanoparticles. Powder Technol. 2004, 145, $20-24$.

(34) Hwang, W. L.; Chen, M.; Cronin, B.; Holden, M.; Bayley, H. Asymmetric Droplet Interface Bilayers. J. Am. Chem. Soc. 2008, 130, 5878-5879.

(35) Chiantia, S.; Schwille, P.; Klymchenko, A. S.; London, E. Asymmetric GUVs Prepared by MbCD-Mediated Lipid Exchange: An FCS Study. Biophys. J. 2011, 100, L01-L03.

(36) Hu, P. C.; Li, S.; Malmstadt, N. Microfluidic Fabrication of Asymmetric Giant Lipid Vesicles. ACS Appl. Mater. Interfaces 2011, 3, 1434-40.

(37) Richmond, D. L.; Schmid, E. M.; Martens, S.; Stachowiak, J. C.; Liska, N.; Fletcher, D. A. Forming Giant Vesicles with Controlled 
Membrane Composition, Asymmetry, and Contents. Proc. Natl. Acad. Sci. U. S. A. 2011, 108, 9431-6.

(38) Matosevic, S.; Paegel, B. M. Layer-by-Layer Cell Membrane Assembly. Nat. Chem. 2013, 5, 958-63.

(39) Meeuwissen, S. A.; Kim, K. T.; Chen, Y.; Pochan, D. J.; van Hest, J. C. M. Controlled Shape Transformation of Polymersome Stomatocytes. Angew. Chem., Int. Ed. 2011, 50, 7070-7073.

(40) Helfrich, W. Elastic Properties of Lipid Bilayers: Theory and Possible Experiments. Z. Naturforsch. 1973, 28c, 693-703.

(41) Berger, O.; Edholm, O.; Jähnig, F. Molecular Dynamics Simulations of a Fluid Bilayer of Dipalmitoylphosphatidylcholine at Full Hydration, Constant Pressure, and Constant Temperature. Biophys. J. 1997, 72, 2002-2013.

(42) Fuchs, P. F.; Hansen, H. S.; Hünenberger, P. H.; Horta, B. A. C. A GROMOS Parameter Set for Vicinal Diether Functions: Properties of Polyethyleneoxide and Polyethyleneglycol. J. Chem. Theory Comput. 2012, 8, 3943-3963.

(43) Bhat, R; Timasheff, S. N. Steric Exclusion is the Principal Source of the Preferential Hydration of Proteins in the Presence of Polyethylene Glycols. Protein Sci. 1992, 1, 1133-1143.

(44) Devanand, K.; Selser, J. C. Asymptotic Behavior and LongRange Interactions in Aqueous Solutions of Poly(ethylene oxide). Macromolecules 1991, 24, 5943-5947.

(45) Wu, E. L.; Cheng, X.; Jo, S.; Rui, H.; Song, K. C.; DavilaContreras, E. M.; Qi, Y.; Lee, J.; Monje-Galvan, V.; Venable, R. M.; et al. CHARMM-GUI Membrane Builder toward Realistic Biological Membrane Simulations. J. Comput. Chem. 2014, 35, 1997-2004. 